\title{
HACIA UNA EXPLOTACIÓN DE LA ENCUESTA \\ DE ESTRUCTURA SALARIAL EN HOSTELERÍA \\ DESDE EL ANÁLISIS EXPLORATORIO DE DATOS CON \\ APLICACIÓN A LA BRECHA SALARIAL DE GÉNERO
}

\author{
Antonio Jesús Acevedo Blanco* \\ Violante Martínez Quintana** \\ UNED
}

\section{RESUMEN}

Este trabajo indaga las posibilidades que presenta la Encuesta Cuatrienal de Estructura Salarial (EES), especialmente para los estudios económicos y sociológicos en el sector del turismo, utilizando el análisis exploratorio de datos (AED) de la estructura salarial en la Hostelería. El artículo se divide en dos partes diferenciadas; en primer lugar, se repasan las instrucciones y la estructura de los datos para la explotación correcta de la encuesta. En segundo lugar, se realiza un ejercicio de AED en el sector económico hostelero donde se testan las diferencias salariales y geográficas, por sectores económicos, cohortes de edad y sexo. Finalmente, tras el análisis efectuado, se concluye poniendo de relieve las grandes posibilidades analíticas que puede desarrollar la base de datos de la encuesta, y se ofrecen algunas líneas de análisis confirmatorias que coadyuvan a complementar esta introducción exploratoria de la brecha salarial.

Palabras clave: Encuesta Cuatrienal Estructura Salarial (EES), salarios, brecha salarial, análisis exploratorio de datos (AED), Hostelería.

\section{TOWARDS AN EXPLOITATION OF THE SALARY STRUCTURE SURVEY IN HOSPITALITY FROM THE EXPLORATORY DATA ANALYSIS WITH APPLICATION TO THE GENDER SALARY GAP}

\section{Abstract}

This paper reviews the possibilities presented by the Quadrennial Salary Structure Survey (EES), especially for economic and sociological studies in the spanish tourist sector, using exploratory data analysis (EDA) of the salary structure in Hospitality. The article is divided into two different parts, first, the instructions and the structure of the data for the correct exploitation of the survey are reviewed. Second, an EDA exercise is carried out in the hospitality economic sector where salary and geographical differences are tested, by economic sector, age and sex cohorts. Finally, after the analysis carried out, it is concluded by highlighting the great analytical possibilities that the survey database can develop, and some confirmatory lines of analysis are offered that help to complement this exploratory introduction to the wage gap.

Keywords: Quadrennial Salary Structure Survey (EES), Wages, Gender Pay Gap, Exploratory Data Analysis (EDA), Hospitality. 
En este trabajo en primer término se aborda cómo explotar la Encuesta Cuatrienal de Estructura Salarial (en adelante EES_2018) para el sector de la Hostelería y Restauración en España y posteriormente se realiza una aproximación empírica a los salarios del sector económico hostelero enfocando la analítica desde el marco metodológico de trabajo del Análisis Exploratorio de datos (AED). En el artículo se plasman el compendio de los debates suscitados a partir de los resultados de las comunicaciones presentadas los autores en la mesa de trabajo «Turismo, Trabajo y Formación» que tuvo lugar en el III Congreso Internacional de Sociología y Antropología del Turismo (SOCATUR 2020) celebrado con formato online en Santa Cruz de Tenerife (Islas Canarias, España) en el mes de febrero de 2021.

Con una estructura claramente diferenciada en la que se trata un plano estrictamente metodológico en la primera parte y empírico en la segunda mitad, el artículo se plantea en base al objetivo de aspirar a cubrir el vacío detectado de comunicaciones metodológicas en Sociología del Turismo y ramas afines. En este sentido en los epígrafes 1 y 2 , así como en anexos, se presenta una propuesta metodológica enfocada en la explotación con garantías de la EES_2018. Entendemos que este recurso puede resultar de utilidad para científicos sociales, estudiantes, técnicos de turismo y otros colectivos profesionales en la obtención de conocimiento del sector económico hostelero y de restauración. Del mismo modo estamos convencidos de la utilidad de esta herramienta metodológica, pues a través de las instrucciones y las descripciones de la estructura de la base de datos expuestas se pueden abarcar investigaciones en otros campos de conocimiento afines en los que el salario se presente como eje central en sus diversos objetos de estudio.

Por otro lado, a raíz de los debates planteados, surgió la propuesta de examinar detenidamente los resultados alcanzados a partir de la explotación de la encuesta en cuanto a salarios y condiciones laborales en el sector hostelero y restaurador. Las circunstancias descritas de intercambio científico resultaron a contemplar otros ángulos de estudio y se decidió dividir el artículo en dos secciones claramente delimitadas de manera que en los epígrafes 3 y 4 se diera cuenta de los resultados obtenidos conforme al AED de la EES_2018.

En resumen, se trata con este artículo de proveer de una herramienta para uso práctico de investigadores en ciencias sociales estructurada en base a dos aproximaciones conexionadas en un solo texto integrador, en la que en la primera parte se presenta una propuesta metodológica detallada para explotación de la EES_2018, y en la segunda, se constata mediante el AED de la encuesta las brechas salariales regionales, de género y edad en el sector de la Hostelería.

*E-mail: aacevedo13@alumno.uned.es. Escuela Internacional de Doctorado de la UNED.

** E-mail:vmartin@poli.uned.es. UNED. 


\section{PROPUESTA PARA EXPLOTAR LA ENCUESTA DE ESTRUCTURA SALARIAL EN HOSTELERÍA}

La principal fuente de ingresos de las personas son las rentas obtenidas del trabajo. En las sociedades salariales (Albarracín, 2003) la ganancia salarial se conforma como el núcleo a partir del cual pivotan la estructuras ocupacionales de los diferentes sectores económicos. En el estudio de los salarios interesa conocer desde la perspectiva sociológica no solo su importe, sino también su distribución, las diferencias entre trabajadores y trabajadoras, la estructura ocupacional y la distribución territorial, entre otras dimensiones analíticas sustanciales. La explotación de la Encuesta Cuatrienal de Estructura Salarial (en adelante EES_2018) brinda una herramienta de gran valor para la investigación de las condiciones laborales, formativas y salariales en el sector hostelero, pues posibilita acceder a los datos recogidos de forma individual (microdatos) gracias a la desagregación que facilita la encuesta por sectores de la Clasificación Nacional de Actividades Económicas (CNACE).

En España la metodología cuantitativa en Turismología acumula una importante tradición de producción científica (Huete, 2014). Sin embargo, el uso de bases de datos secundarias, no exclusivamente centradas en actividades turísticas, no está lo suficientemente desarrollado como en el caso de la investigación académica cuando trabaja con encuestas o registros construidos exprofeso. De aquí que en este trabajo se proponga en primer lugar ilustrar los procedimientos, las instrucciones básicas y la estructura de la base de datos de forma que pueda abordarse la EES_2018 con plenas garantías. La encuesta no presenta una especial dificultad para su explotación; sin embargo, requiere disponer de unos apuntes básicos de la estructura y de la recogida de datos. En este sentido, resulta especialmente recomendable enfocar estas orientaciones en el conocimiento del marco muestral de la encuesta, la extracción de datos, la ponderación y la elevación de estos. Estas recomendaciones y apuntes metodológicos resultarán sin duda útiles para aquellos turismólogos poco familiarizados con la explotación de grandes bases de datos, pues habitualmente no se suelen tener presentes estas consideraciones metodológicas en el tratamiento de los microdatos, y la investigación podría llegar a obtener resultados inexactos.

Tras un repaso de la bibliografía científica más relevante en la segunda parte del artículo se propone un ejercicio estadístico exploratorio de datos de la estructura salarial y ocupacional en hostelería. La utilidad central del AED radica en las múltiples posibilidades que ofrece para cimentar proyectos de investigación, marcos de análisis y modelos teóricos a partir de grandes conjuntos de datos (Salvador y Gargallo, 2003). Olivares (2002) señala que el principal potencial de los procedimientos de AED se enfoca principalmente en la búsqueda de patrones no esperados en los datos, o, dicho de otra forma, el AED es un conjunto de técnicas estadísticas y gráficas orientadas a revelar esquemas en las variables o conjuntos de variables con la orientación principal de iniciar procesos analíticos de prueba de hipótesis y/o construcción de teorías emergentes.

La analítica exploratoria es una técnica de gran recorrido en ciencias sociales. Desde la aportación de Tukey (1997) hasta la actualidad, el AED ha ido cimentando 
un amplio desarrollo, que en gran medida se ha visto acelerado por los progresos en computación y gestión de grandes bases de datos. Como plantea Escobar (1999) gracias al auge de los grandes bancos de datos el AED, más que una técnica o táctica de investigación, se propone como paradigma y marco de trabajo en Sociología. En este sentido, De Rada (2002) sostiene que el primer paso de toda investigación cuantitativa sociológica debe comenzar con una introducción descriptiva de los datos y su estructura. Los estadísticos descriptivos de frecuencias, de posición en las distribuciones, el análisis de las medias, y el análisis gráfico, componen el aparataje metodológico básico para el desarrollo del AED.

El marcado carácter inductivo del artículo se complementa con una revisión de la literatura científica para las dimensiones de análisis estudiadas. El objetivo que se persigue, y principal aportación de este trabajo, es doble. En primer lugar, presentamos un acercamiento metodológico, que, de cara a la investigación sociológica en Turismo, puede ser de utilidad para introducirse con garantías en el conocimiento de las estructuras salariales y ocupacionales del sector turístico. Para este fin, además del cuerpo textual del artículo, donde se abordan las características básicas y estructura de la EES, se adjuntan en el anexo I las instrucciones precisas en archivo de sintaxis para que utilizando software estadístico estándar se logre segmentar el archivo por el campo de ocupación de la hostelería y restauración, elevar y ponderar la muestra y los procesos e instrucciones necesarias para crear variables derivadas como salario anual, salario mensual o salario/hora, clave este último para la comparabilidad entre asalariado y asalariadas, y construcción de indicadores de brecha salarial de género.

En segundo lugar, se testan las diferencias de salarios entre sectores económicos realizando las pruebas necesarias para comprobar la significatividad de las diferencias de medias. Posteriormente, enfocados en el sector hostelero y restaurador se utiliza un procedimiento similar, y se testan los salarios en hostelería, las diferencias salariales dentro de los mercados locales de trabajo en Espańa, y las diferencias salariales entre edad y sexo. En síntesis, se propone un planteamiento inductivo en cuanto a la generación de teoría ligada a la explotación de la base de datos mediante un ejercicio de AED. En este sentido, tanto la línea metodológica como las evidencias empíricas expuestas se orientan a futuros desarrollos y proyectos de investigación confirmatorios, que el equipo de investigación autor de este trabajo desarrolla actualmente en colaboración académica multidisciplinar.

\section{LA ENCUESTA CUATRIENAL DE ESTRUCTURA SALARIAL (EES_2018)}

El INE pone a libre disposición de investigadores y profesionales una amplia gama de encuestas con ficheros de microdatos asociados, o desagregados a nivel de respuesta individual. En efecto, la ventaja de trabajar con microdatos permite llevar a cabo análisis flexibles, relacionar variables y realizar operaciones estadísticas, que no siempre están disponibles en las aplicaciones online del Instituto Nacional de Estadística (INE). 
Entre estos productos estadísticos gratuitos se encuentra la EES_201811, que, a diferencia de otras bases de datos con temáticas similares, por ejemplo, la Encuesta de Población Activa (EPA), permite la desagregación del archivo de microdatos en base a la CNACE. Aplicando la operación de segmentar el archivo por la variable $\mathrm{CNACE}^{2}$ correspondiente al sector de la Hostelería (código I, ver tabla 1) se accede a una amplia gama de variables de gran utilidad para la investigación salarial, laboral y de condiciones de trabajo en la actividad económica hostelera, sector que se conforma como el principal escenario profesional del turismo como industria.

\begin{aligned} & \hline \multicolumn{1}{c}{ T } \\ & \hline 55 Servicios de alojamiento \\ & \hline 551 Hoteles y alojamientos similares \\ & \hline 5510 Hoteles y alojamientos similares \\ & \hline 552 Alojamientos turísticos y otros alojamientos de corta estancia \\ & \hline 5520 Alojamientos turísticos y otros alojamientos de corta estancia \\ & \hline 553 Campings y aparcamientos para caravanas \\ & \hline 5530 Campings y aparcamientos para caravanas \\ & \hline 559 Otros alojamientos \\ & \hline 5590 Otros alojamientos \\ & \hline 56 Servicios de comidas y bebidas \\ & \hline 561 Restaurantes y puestos de comidas \\ & \hline 5610 Restaurantes y puestos de comidas \\ & \hline 562 Provisión de comidas preparadas para eventos y otros servicios de comidas \\ & \hline 5621 Provisión de comidas preparadas para eventos \\ & \hline 5629 Otros servicios de comidas \\ & \hline 563 Establecimientos de bebidas \\ & \hline 5630 Establecimientos de bebidas \\ & \hline\end{aligned}

Según lo previsto en el Reglamento del Consejo 530/1999 la EES_2018 es una encuesta cuatrienal elaborada dentro del marco europeo estadístico, que viene realizándose en España con igual metodología desde 2010, y desde el año 2002 con unos criterios metodológicos y de contenido comunes en el marco de la Unión Europea (UE) para obtener unos resultados comparables sobre el nivel, la estructura y distribución del salario entre sus Estados miembros. Los países facilitan por medio

${ }^{1}$ https://www.ine.es/dyngs/INEbase/es/operacion.htm?c=Estadistica_C\&cid=125473 $6177025 \&$ menu=resultados\&idp=1254735976596\#!tabs-1254736195110.

${ }^{2}$ https://www.ine.es/dyngs/INEbase/es/operacion.htm?c=Estadistica_C\&cid=12547361 77032 \&idp $=1254735976614$. 
de la EES cuatrienalmente datos sobre ingresos, número de empleados y actividad económica, basándose en un acuerdo entre Eurostat y los institutos estadísticos de los Estados miembros. De esta forma se proporciona información comparable a escala de la UE sobre las relaciones entre salarios, características individuales de los trabajadores y trabajadoras (sexo, edad, ocupación, nivel educativo, antigüedad) y empleadores y empleadoras. La amplitud de la muestra, su periodicidad y la amplia gama de variables que recoge hacen de la EES_2018 una herramienta muy útil, no solo para la explotación estadística socioeconómica comparada, sino también para investigaciones que abarquen dimensiones de estudio sociológicas, de estudios regionales, de género o ciencias del trabajo, entre otras.

En cuanto a las características de la encuesta y el muestreo tenemos que la encuesta, subdivide la muestra de la EES_2018 por zonas geográficas, abarcando la totalidad del territorio estatal espańol de manera desagregada en los microdatos y por Nomenclaturas de la Unidades Territoriales Estadísticas (NUTS1). Para el caso de España abarcan unidades territoriales superiores a las comunidades autónomas. El marco muestral poblacional está compuesto de trabajadores y trabajadoras por cuenta ajena, que presten sus servicios en centros de cotización y se encuentren de alta en la Seguridad Social durante todo el mes de octubre del año en que se realiza la encuesta.

La encuesta se realiza en el mes de octubre del año de referencia, año 2018 para nuestro caso. Por una parte, este mes tiene la ventaja de considerarse normal en cuanto a verse poco afectado tanto por la estacionalidad productiva como por pagos salariales extraordinarios. Por otra parte, en octubre el sector hostelero comienza la temporada baja especialmente para destinos de sol y playa. Esta particularidad estacional de la EES_2018 supone una limitación de la base de datos que debe ser tenida en cuenta para aquellas investigaciones enfocadas principalmente desde el turismo.

Para realizar la EES_2018 se parte del Registro General de Cuentas de Cotización a la Seguridad Social actualizado a 31 de octubre del año de referencia. Del registro se obtiene el Directorio de Cuentas de Cotización a la Seguridad Social que clasifica las divisiones de actividades económicas objeto de estudio. Este directorio sirve de base para extraer las muestras de cuentas de cotización. El procedimiento de selección aleatoria de unidades corresponde a un muestreo bietápico estratificado, donde las unidades de primera etapa son las cuentas de cotización a la Seguridad Social, mientras que las de segunda etapa son los trabajadores y las trabajadoras.

Las unidades de primera etapa se clasifican según su actividad económica de acuerdo con la clasificación nacional vigente que se consideran poblaciones independientes a efectos del muestreo. Cada actividad se estratifica por comunidades autónomas (18 en total, considerando el conjunto formado por Ceuta y Melilla) y por ocho intervalos de tamaño, que son los siguientes:

1. De 1 a 4 trabajadores.

2. De 5 a 9 trabajadores.

3. De 10 a 19 trabajadores.

4. De 20 a 49 trabajadores.

5. De 50 a 99 trabajadores. 
6. De 100 a 199 trabajadores.

7. De 200 a 499 trabajadores.

8. Más de 500 trabajadores.

La enumeración de unidades seleccionadas en la primera etapa se remite de nuevo a la Tesorería General de la Seguridad Social, que obtiene la lista de trabajadores que estuvieron de alta durante todo el mes de octubre del año de referencia. A partir de este directorio se seleccionan los trabajadores, y las unidades de segunda etapa. En cuanto al código I de la CNACE (hostelería) la muestra la componen 8192 unidades, un $3.2 \%$ de la muestra total sin ponderar. Esta muestra se eleva a 19514 registros suponiendo un $9 \%$ del marco muestral cuando realizamos las operaciones de ponderación que posteriormente se desarrollan.

\section{Muestra de La encuesta, PONDERACión y ELEVACión}

El diseño de la EES_2018 no es proporcional para todos los casos o, en otros términos, todas las repuestas individuales no tienen el mismo peso poblacional en cuanto a los resultados totales. La EES_2018 incluye en su diseño la variable FACTOTAL, que actúa como factor de elevación de la muestra asignando un valor numérico a cada uno de los casos individuales. Conforme al peso asignado, cuando se pondera el archivo por el factor de elevación cada caso individual pasa a contar como varios individuos poblacionales.

En la tabla 2 se presentan las frecuencias y porcentajes de la variable REGULACIÓn, que hace referencia a la forma de clasificación de las relaciones laborales principalmente en cuanto al ámbito de aplicación de los convenios colectivos. En esta tabla se observa cómo el total de la muestra asciende a 216726 registros sin elevar la muestra. Una vez elevada la muestra, las frecuencias absolutas ascienden a 12976074 casos. Para realizar procedimientos inferenciales el factor de elevación no permite un buen uso pues se requiere un factor de ponderación que reequilibre la muestra de forma que los cálculos se realicen sobre el tamaño muestral válido según el diseño de encuesta planteado.

Siguiendo el procedimiento empleado para la explotación de la EPA podemos hallar un factor de ponderación que reequilibre la muestra teniendo en cuenta la relación de pesos muestrales y poblacionales. Si $w_{i}$ resulta la cantidad de entrevistados en el estrato muestral y $w$ el tamaño de la muestra, $W_{i}$ la cantidad de personas en el estrato poblacional y $W$ el tamańo de la población, se mantiene la siguiente proporción entre el tamaño de cada estrato y el tamaño de la población.

$$
\frac{w_{i}}{w}=\frac{W_{i}}{W}
$$

Despejando obtenemos la fórmula para hallar el factor de ponderación

$$
w_{i}=\frac{W_{i}}{W} w
$$


Tomando nota del total de la muestra sin ponderar $(w=216726)$ y elevada $(W=12976074)$ podemos calcular el factor de ponderación necesario para nivelar proporcionalmente la muestra. Con la orden COMPUTE en el comando de sintaxis la fórmula para crear una variable de ponderación será:

\section{COMPUTE PONDERA = FACTOTAL*216726/12976074.}

Realizado este cálculo podemos observar en la tabla 2 que en la columna de frecuencias se indica el número de entrevistas realmente realizadas, la segunda columna eleva estas frecuencias y estima el número total de personas que pertenecen a esta población y por último la tercera columna pondera la muestra de forma que la reequilibra teniendo en cuenta la proporción de personas que realmente pertenecen a cada categoría en base al diseño muestral planteado.

\section{TABLA 2. FRECUENCIAS DE ENCUESTADOS POR LA EES ELEVADAS Y PONDERADAS}

\begin{tabular}{|c|c|c|c|c|c|c|}
\hline & 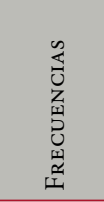 & 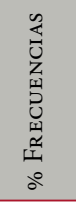 & 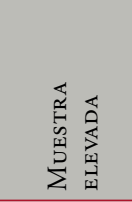 & 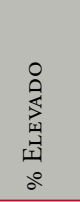 & 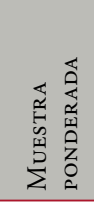 & 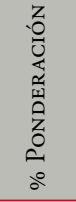 \\
\hline Estatal sectorial & 68490 & 31,6 & 3757796 & 29,0 & 62763 & 29,0 \\
\hline $\begin{array}{l}\text { Sectorial de ámbito inferior } \\
\text { (Autonómico, provincial o comarcal) }\end{array}$ & 74733 & 34,5 & 5565271 & 42,9 & 92951 & 42,9 \\
\hline De empresa o grupo de empresas & 44624 & 20,6 & 1910685 & 14,7 & 31912 & 14,7 \\
\hline De centro de trabajo & 7485 & 3,5 & 324627 & 2,5 & 5422 & 2,5 \\
\hline Otra forma de regulación & 21394 & 9,9 & 1417695 & 10,9 & 23678 & 10,9 \\
\hline Total & 216726 & 100,0 & 12976074 & 100,0 & 216726 & 100,0 \\
\hline
\end{tabular}

Fuente: elaboración propia con SPSS 25 a partir de EES_2018.

Para ilustrar el procedimiento realizado conviene revisar la tabla 2 resaltando en primer término las columnas totales que corresponden al total de entrevistados por una parte (frecuencias y frecuencias ponderadas), y al total de la población empleada en el sector hostelero por la otra (columna de frecuencias elevadas). Como podemos comprobar las columnas de porcentajes coinciden los marginales de cada grupo en cuanto a frecuencias elevadas y ponderadas. Esta igualdad de porcentajes de cada categoría se debe al reequilibrio muestral que se produce cuando ponderamos la EES_2018 por el factor de ponderación o elevación. Si prestamos atención a la primera fila la regulación laboral sectorial de ámbito estatal supone un 31.6\% de los realmente entrevistados por la EES_2018; sin embargo, este porcentaje disminuye al 29\% si tenemos en cuenta el total de trabajadores acogidos a esta regulación o, en otras palabras, a la muestra elevada. De igual forma el $29 \%$ corresponde al porcentaje ponderado tras reequilibrar la muestra conforme al diseño muestral propuesto. 
Hay que tener presente que «la base de datos siempre debe trabajarse como base ponderada", bien por el factor de ponderación (PONDERA) cuando realicemos operaciones de estadística inferencial, o por el factor de elevación (FACTOTAL) cuando los procedimientos realizados correspondan a operaciones de estadística descriptiva.

\section{CREACIÓN DE VARIABLES DERIVADAS}

En esta sección ofrecemos los recursos necesarios para crear las variables derivadas no presentes en el archivo de microdatos, pero de gran importancia clave para la investigación salarial y la obtención de resultados eficientes. En el anexo 1 de este artículo se reproduce la sintaxis necesaria para realizar los procedimientos de creación de variables derivadas, tanto de las de ganancias salariales como las de tiempo de trabajo.

En primer lugar, con respecto a las variables salariales, la variable de ganancia por hora (SALHORA) resulta de especial interés pues permite cotejar el salario/ hora entre trabajadores con diferentes jornadas, completas o parciales. La ganancia por hora se calcula dividiendo el salario obtenido en el mes de referencia entre las horas trabajadas, tanto horas de trabajo normales como extraordinarias. Este indicador resulta especialmente relevante para calcular la brecha salarial de género no ajustada que, sintéticamente expresada, representa la diferencia entre los ingresos brutos medios por hora de hombres y mujeres como porcentaje de los ingresos brutos medios por hora de los empleados remunerados hombres. La metodología empleada en la EES aporta en el marco de la UE el procedimiento estandarizado necesario para la construcción del indicador de brecha salarial de género, por lo que resulta especialmente relevante desarrollar adecuadamente los procesos necesarios para la obtención del índice en la investigación salarial en general, la Turismología en particular y los estudios de género, entre otras disciplinas.

Las variables de ganancia mensual resultan también especialmente relevantes ofreciendo grandes posibilidades analíticas pues debemos tener en cuenta que el periodo de referencia retributivo por excelencia en Espańa y la mayor parte de la UE es el mes. En la EES_2018 se construyen tres variables para las ganancias mensuales: SALMOR, que recoge los pagos mensuales ordinarios sin incluir pagas extras; SALMES, que incluye el salario mensual total recogiendo pagos extraordinarios y pagos por horas extra, y por último la variable SALNETO, que recoge el salario neto descontando al salario mensual el Impuesto a la Renta para Personas Físicas (IRPF) y cotizaciones a la Seguridad Social. Con respecto a los trabajadores que no han obtenido un salario mensual completo por ausencias no remuneradas se calcula la variable teniendo en cuenta los días de salario completo. De esta forma se permite la comparabilidad de ganancias mensuales entre trabajadores.

Por su parte, con respecto a las ganancias anuales se recogen tanto los devengos extraordinarios en la variable SALANUAL como el salario anual neto (SALOR), denotado como salario anual ordinario que no incluye pagos extraordinarios ni los pagos en especie. Del mismo modo que en las ganancias mensuales para obtener 
ganancias anuales comparables, se ajusta el salario de aquellos trabajadores que no permanecieron todo el año en el centro de trabajo. Para ello se les asigna un salario anual equivalente al que hubieran percibido si hubieran estado trabajando durante todo el año.

Las variables de tiempo de trabajo son desde el punto de vista estadístico de las más complicadas de obtener. Internacionalmente se adopta el consenso de calcular adicionando a las horas realizadas en la jornada habitual de trabajo las horas extraordinarias. A esta suma hay que restar las horas no trabajadas por absentismo, por ser un tiempo de trabajo perdido (Martínez-Quintana, 1994). La EES_2018 no se orienta al estudio exhaustivo del tiempo de trabajo, por lo que adopta una solución común para la Unión Europea que consiste en considerar para los cálculos únicamente el absentismo cuando este afecte al salario. De esta forma, si las ausencias afectaron al salario se toma la parte salarial no afectada por el absentismo ajustándose al periodo de referencia completo. En el caso de que el absentismo no afecte al salario, como pueda ser el caso de vacaciones, permisos retribuidos o salidas sindicales, se considera que se realiza la jornada habitual, ya que el salario corresponde a un mes normal de trabajo.

\section{REVISIÓN BIBLIOGRÁFICA}

En los análisis de salarios en sectores retributivamente deprimidos se apunta que los empleos pueden clasificarse en torno a los niveles salariales en los que se inscriben sus sectores económicos, independientemente de la productividad marginal del negocio. Esta clasificación salarial implica dividir los empleos entre «buenos» y «malos» atendiendo al estatus social otorgado por la retribución económica, a la que además deben añadirse otros factores como la penosidad, la festividad, la turnicidad o el esfuerzo requerido para ejecutar estos, en algunos casos, subempleos (Acemoglu, 2019).

En el sentido indicado parece lógico pensar que ocupar salarialmente las últimas posiciones en el ranking de las ocupaciones profesionales indica taxativamente un alto nivel de segregación ocupacional de los colectivos asalariados. Resulta por otra parte comprensible especular que si, además de precario, el sector hostelero se propone como una ocupación "mal pagada», los atractivos para emprender carrera laboral en hostelería se reducen considerablemente (Martínez-Gayo, 2019).

La principal variable objeto de investigación salarial en el sector turístico y especialmente en clave de brecha salarial ha sido el género, revisado en profundidad por varios autores, entre ellos Cueto y Sánchez-Sánchez (2006), Conde-Ruiz y Marra de Antínano (2016) o Rodríguez-González (2018). Desde un enfoque geográfico los aportes de Rivera Mateos (2018) ańaden a la perspectiva de género la dimensión rural como otra vuelta de tuerca en el análisis de los déficits salariales. Otra línea de trabajo empírico salarial en el sector hostelero tiene que ver con las diferencias salariales de género en cuanto al déficit formativo y de capital social del sector, línea propuesta por Ons-Cappa et al. (2017) utilizado modelos de regresión lineal multivariante. En este sentido el trabajo de Pozo-García et al. (2011) comple- 
menta el análisis salarial regional con la ya indicada segregación ocupacional hostelera producida por los déficits formativos de su cuerpo salarial.

Especialmente relevantes en este campo han sido las investigaciones de Huete et al. (2016), donde se presentan evidencias de la brecha salarial entre sexos, la segregación vertical y la mayor precariedad laboral que sufren las mujeres en el subsector de la hostelería en Espańa. En el mismo sentido el informe de Moreno y Cañada (2018) sobre cómo se ha abordado el trabajo de las mujeres en el sector turístico constituye un referente fundamental para entender las segregaciones verticales y horizontales producidas. Martínez-Quintana y Martínez-Gayo (2019) en similar línea aportan las claves para entender el proceso de desigualdad laboral entre sexos en el sector turístico aportando una revisión comparativa salarial con la Encuesta Anual de Estructura Salarial a partir del estudio de salarios con la aplicación online del INE. Por último, Ramos et al. (2002), con una muestra propia construida en una economía especializada en turismo como Baleares, analizan empírica y específicamente las diferencias salariales en el sector hostelero, donde afirman, entre otros, la existencia de una clara discriminación retributiva en hostelería por razones de género.

\section{PROCEDIMIENTO, RESULTADOS Y DISCUSIÓN}

Para situar el análisis, en las siguientes secciones, nos adentramos en la estructura salarial del sector hostelero y de la restauración mediante un ejercicio de $\mathrm{AED}$ en el que se realizan los siguientes procedimientos:

- En primer lugar, se examina el lugar que ocupa el sector hostelero dentro del ranking salarial entre sectores de la CNACE.

- Posteriormente se revisa la estructura salarial en hostelería desde la óptica exploratoria mostrando los principales estadísticos descriptivos de los indicadores salariales más relevantes (salario hora, mensual y anual).

- En España la industria turística es un sector productivo muy heterogéneo, diverso y especializado. En este sentido geográficamente las diferencias salariales entre mercados turísticos importan, por lo que se requiere enfocar el análisis en base a comprobar la significatividad de estas diferencias.

- Para finalizar la aproximación empírica centramos el procedimiento analítico en torno a las características individuales del cuerpo laboral asalariado en hostelería. Se revisa la estructura salarial en base al sexo y edad de los sujetos situando el eje en las diferencias retributivas entre estratos poblacionales del sector.

Para comprobar la significatividad de las diferencias entre medias se realizan para cada una de las dimensiones analíticas procedimientos de análisis de la varianza (López-Roldán y Fachelli, 2018) para un factor (ANOVA) y análisis de la covarianza (ANCOVA) cuando comprobamos las diferencias entre varios factores (edad y sexo). Se propone en todos los supuestos la modelización estadística en forma de aceptación o rechazo de la hipótesis nula de igualdad de medias planteán- 


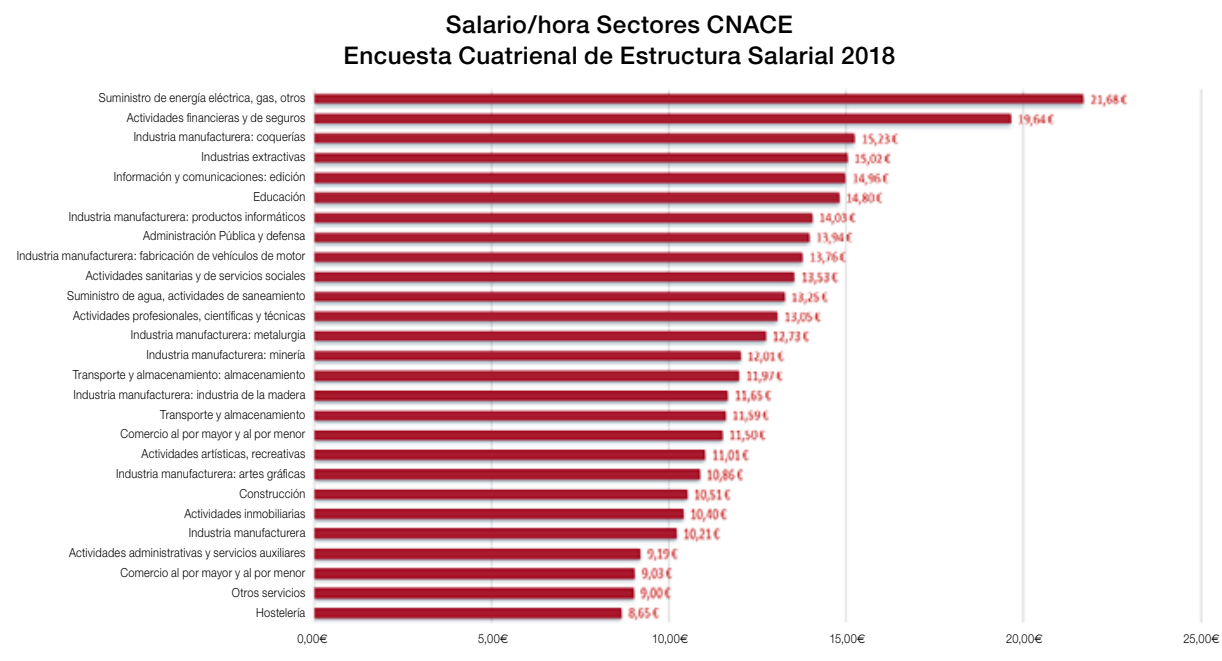

Gráfico 1: salario/hora entre sectores CNACE.

Fuente: elaboración propia con SPSS 25 a partir de EES_2018.

dose, en su caso, la hipótesis alternativa que asume la existencia de condicionantes, patrones o estructuras en los datos que de alguna forma afectan a la proposición de igualdad de medias salariales (Almazán et al., 2011).

\section{SALARIOS ENTRE SECTORES}

El procedimiento de análisis de la estructura salarial en Hostelería se inicia con un gráfico ordenando de mayor a menor las ocupaciones según el salario hora. Una rápida visualización del gráfico 1 nos ayuda a situar el lugar en la escala retributiva que el sector hostelero ocupa dentro de la CNACE. Podemos comprobar que la hostelería y restauración ocupa la última de las posiciones en la escala retributiva no superando la barrera de los $9 €$ por hora de trabajo realizado.

En el cálculo del salario hora el supuesto de la homogeneidad de varianzas entre grupos resulta significativo. Por su parte, resulta igualmente significativa la prueba del análisis de la varianza (ANOVA) para la comparación entre grupos por salario hora, o lo que viene a ser lo mismo, las diferencias de salarios hora entre grupos de la CNACE resultan estadísticamente significativas y, por tanto, con una alta probabilidad puede atribuirse que las diferencias salariales entre sectores económicos no se deben al azar. Igualmente se comprueba la significatividad en la diferencia de medias para los salarios mensuales y anuales resultando positivo el supuesto (tabla 4).

Los procedimientos ANOVA proponen la significatividad, o no, de las diferencias de medias entre grupos. Lo que no se indica con la ANOVA es entre qué grupos existe significatividad en las diferencias. Para comprobar este supuesto se 


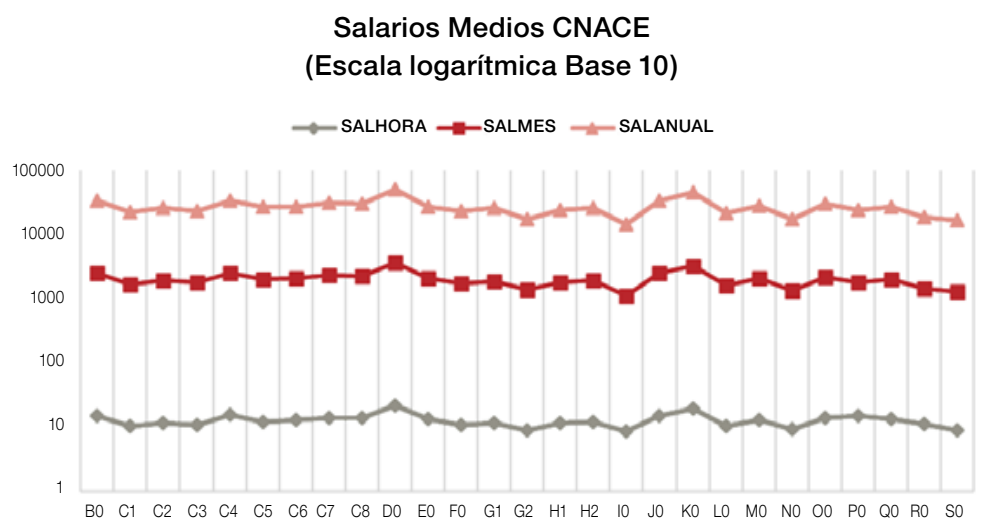

Gráfico 2: salarios medios CNACE en escala logarítmica.

Fuente: elaboración propia con Excel a partir de EES_2018.

utiliza el procedimiento DHS (Honestly Significant Difference) de Tukey. Aplicando el procedimiento se comprueba cómo existe significatividad en las diferencias de medias entre el sector de la hostelería (I0) con respecto al resto de sectores que componen la CNACE.

\begin{tabular}{lccc}
\hline \multicolumn{4}{c}{ TABLA 3. SUBCONJUNTOS HOMOGÉNEOS HSD DE TUKEY } \\
\hline SALHORA: subconjuntos homogéneos & CNACE & N & Subconjunto para alfa $=0.05$ \\
\hline HSD Turkey a,b & I0 & 19514 & $8,6497 €$ \\
\hline $\begin{array}{l}\text { Hostelería: servicios de alojamiento, servicios de } \\
\text { comidas y }\end{array}$ & S0 & 4845 & $8.9978 €$ \\
\hline $\begin{array}{l}\text { Otros servicios: actividades asociativas, repara- } \\
\text { ción de ordenadores }\end{array}$ & G2 & 21384 & $9,0297 €$ \\
\hline $\begin{array}{l}\text { Comercio al por mayor y al por menor; repara- } \\
\text { ción de vehículo }\end{array}$ & N0 & 17927 & $9,1857 €$ \\
\hline Actividades administrativas y sevicios auxiliares
\end{tabular}

Fuente: elaboración propia con SPSS 25 a partir de EES_2018 2.

Para seguir profundizando en el AED de la estructura salarial del resultado de los subconjuntos homogéneos (tabla 3) se extraen los grupos ocupacionales que mayor similitud tienen en cuanto a salario hora con el sector hostelero. Los resultados proponen una estructura salarial para el sector hostelero similar a los sectores del comercio y servicios.

Para finalizar esta sección en el gráfico 2 se estandarizan los resultados en logaritmo base 10 para de esta forma homogeneizar la escala y facilitar la comparabilidad de los devengos salariales más habituales. Hay que tener en consideración que el periodo más habitual en España para el cobro de salarios es el mes, si bien 


\begin{tabular}{|c|c|c|c|c|c|c|}
\hline & & SUMA DE CUADRADOS & Gl & $\begin{array}{c}\text { Media } \\
\text { CUADRÁtica }\end{array}$ & $\mathrm{F}$ & Sig. \\
\hline \multirow{3}{*}{ SALHORA } & Entre grupos & 1382546,308 & 26 & 53174,858 & 1108,086 &, 000 \\
\hline & Dentro de grupos & 10398911,694 & 216698 & 47,988 & & \\
\hline & Total & 11781458,001 & 216724 & & & \\
\hline \multirow{3}{*}{ SALMES } & Entre grupos & 43376016821,907 & 26 & 1668308339,304 & 1280,001 &, 000 \\
\hline & Dentro de grupos & 282436540688,937 & 216698 & 1303364,778 & & \\
\hline & Total & 325812557510,844 & 216724 & & & \\
\hline \multirow{3}{*}{ SALANUAL } & Entre grupos & 9098199010503,791 & 26 & 349930731173,223 & 1336,919 &, 000 \\
\hline & Dentro de grupos & 56719413649476,830 & 216698 & 261744056,934 & & \\
\hline & Total & 65817612659980,620 & 216724 & & & \\
\hline
\end{tabular}

Fuente: elaboración propia con SPSS 25 a partir de EES_2018.

el salario medio por hora se propone como el devengo en menor medida condicionado por el factor tiempo de trabajo y, por tanto, resulta la escala más eficaz para trabajar comparaciones individuales y brechas salariales de género. Por otra parte, cabe destacar que el salario mensual resulta el periodo de devengos salariales más intuitivo y comprensible pues sitúa la estimación salarial con relación a una medida retributiva y socialmente compartida. De igual forma el salario anual resulta una escala de medida salarial muy utilizada especialmente en el cálculo de impuestos.

Teniendo en cuenta las premisas anteriores, el sector hostelero en cada uno de los periodos de devengos salariales continúa ocupando el último de los puestos. Como podrá intuirse el procedimiento ANOVA (tabla 4) tanto para el indicador salario/anual como para el salario/mes resulta significativo. Cabe reseñar que en cuanto a la posible formación de subgrupos homogéneos HSD de Tukey ambos indicadores no conforman ninguna agrupación con otros sectores salarialmente similares. De esta forma se refuerza la hipótesis de diferencias de medias entre sectores a la vez que, en cierta medida, se afianza la situación de última posición retributiva del sector hostelero en el ranking de sectores ocupacionales productivos.

\section{SALARios EN hostelería ${ }^{3}$}

En esta sección se revisan los principales estadísticos descriptivos y medidas de posición de los indicadores salariales en hostelería. El indicador más usual y base para los contrastes de medias es el salario medio o media de la distribución.

\footnotetext{
${ }^{3}$ Se identifican como outliers los siguientes casos: 1522/630/528. Se eliminan de la base de datos por este orden para que no se produzca error en el borrado por fallo en la numeración. Si bien
} 


\begin{tabular}{|c|c|c|c|c|}
\hline \multicolumn{5}{|c|}{ TABLA 5. ESTADÍSTICOS DESCRIPTIVOS DE SALARIOS EN HOSTELERÍA } \\
\hline & & SALMES & SALHORA & SALANUAL \\
\hline Media & & $1124,13 €$ & $8,60 €$ & $14338,05 €$ \\
\hline $95 \%$ de intervalo & \multirow{2}{*}{ Límite inferior } & \multirow{2}{*}{$1141,54 €$} & \multirow{2}{*}{$8,60 €$} & \multirow{2}{*}{$14213,41 €$} \\
\hline de confianza & & & & \\
\hline para la media & Límite superior & $1133,71 €$ & $8,69 €$ & $14462,69 €$ \\
\hline Media recortada al 5\% & & $1075,38 €$ & $8,27 €$ & $13742,81 €$ \\
\hline Mediana & & $1123,00 €$ & $8,04 €$ & $14303,03 €$ \\
\hline Desv. desviación & & $683,05 €$ & $3,31 €$ & $8882,67 €$ \\
\hline Mínimo & & $11,25 €$ & $2,80 €$ & $285,97 €$ \\
\hline Máximo & & $14715,04 €$ & $84,57 €$ & $328240,03 €$ \\
\hline Rango & & $14703,79 €$ & $81,76 €$ & $327954,06 €$ \\
\hline Rango intercuartil & & $816,89 €$ & $2,37 €$ & $10768,01 €$ \\
\hline Asimetría & & $2,34 €$ & $4,79 €$ & $3,50 €$ \\
\hline Curtosis & & $18,13 €$ & $48,93 €$ & $65,04 €$ \\
\hline
\end{tabular}

Fuente: elaboración propia con SPSS 25 a partir de EES_2018.

Se muestra además el indicador de salario medio recortado al 5\% donde se calcula la media del $90 \%$ de la distribución al eliminarse el 5\% de los datos tanto superiores como inferiores. Por su parte el salario mediano, o lo que viene a ser igual, la mediana de la distribución, indica el punto de corte salarial por el cual se divide a los trabajadores en dos partes iguales. Se presentan igualmente los puntos mínimos y máximos de las distribuciones salariales eliminado outliers (casos atípicos), así como otras medidas de forma de las distribuciones como la asimetría ${ }^{4}$ o curtosis ${ }^{5}$ en la que no nos detendremos por razones de espacio pero que por su forma parecen entrever gran concentración de valores en las zonas inferiores de las distribuciones o, en otras palabras, elevada concentración de efectivos laborales con sueldos bajos.

no resulta estrictamente necesario eliminar estos casos, pues los resultados no variarán sustancialmente, si aconsejamos el borrado de los casos para una mayor precisión en los cálculos.

* Identificar casos atípicos.

Detectanomaly

/VARIABLES SCALE=SALMES SALANUAL SALHORA

/Print anomalyList

/HANDLEMisSing APPLY=No

/Criteria pCtanomalouscases=5 Anomalycutpoint=2 Minnumpeers=1 Maxnumpeers=15 NumREASONS $=1$.

${ }^{4}$ Se presentan distribuciones salariales positivas gran número de puntuaciones bajas o escoradas hacia la izquierda.

${ }_{5}$ Se presentan distribuciones salariales leptocúrticas o concentradas en pocos valores. 


\begin{tabular}{|c|c|c|c|}
\hline $\begin{array}{l}\text { SALARIOS MEDIOS } \\
\text { RECORTADOS } 5 \%\end{array}$ & I0 Hostelería & Todos sectores CNACE & BRECHA SALARIAL \\
\hline SALHORA & $8,27 €$ & $11,04 €$ & $-25,09 \%$ \\
\hline SALMES & $1075,38 €$ & $1696,89 €$ & $-36,62 \%$ \\
\hline SALANUAL & $13742,81 €$ & $22463,00 €$ & $-38,82 \%$ \\
\hline
\end{tabular}

Fuente: elaboración propia con SPSS 25 a partir de EES_2018.

Examinando los resultados de la tabla 5 cabe destacar en primer lugar que la mitad de los trabajadores del sector cobran mensualmente con impuestos incluidos un salario inferior a $1123 €$ (8€ por hora o $14303 €$ al ańo). Sin extendernos en demasía en la interpretación sociológica de los resultados, sí cabe reseñar sobre estos que se incide de nuevo en el bajo nivel retributivo del sector apuntado en la sección anterior. El salario medio recortado al $5 \%$ propone una medida más robusta al eliminar para su cálculo los puntos más alejados de la distribución y que, por extremos, puedan distorsionar los resultados. Este indicador se sitúa en $1075 €$ mensuales incidiendo en la segregación salarial apuntada anteriormente donde se revisa con mayor detalle con los resultados de la tabla 5.

En la tabla 6 se calcula la brecha salarial del sector hostelero con respecto al total de grupos que componen la CNACE. Podemos observar la gran diferencia salarial de la hostelería con respecto de otros sectores. En términos absolutos llaman la atención las diferencias de retribuciones de más de $600 €$ de sueldo mensual con respecto de la media de la CNACE. La brecha salarial ocupacional asciende al 25,09\% por hora de trabajo realizada, o lo que viene a ser igual, un trabajador del sector hostelero y de restauración cobra de media por hora trabajada una cuarta parte menos que el salario medio por hora general.

Este déficit salarial se ve acrecentado hasta el 36,62\% cuando realizamos los cálculos para el salario mensual. Asciende por su parte la brecha salarial al 38,82\% cuando el cálculo retributivo se realiza en base a un periodo de tiempo anual. Estas diferencias salariales en periodos retributivos socialmente estandarizados en España vienen a reiterar otro aspecto fundamental a la hora de entender el trabajo hostelero, su marcado carácter estacional. Es lógico pensar que, si existen diferencias en la brecha salarial ocupacional de más de un 13\% entre salario hora y salario anual, estas diferencias se deberán al menor número de días trabajados en hostelería con respecto a las jornadas de trabajo realizadas en el resto de los sectores.

Para ampliar la explicación en el sentido indicado el gráfico 3 muestra la estructura ocupacional por sexo del sector de la hostelería. Los acrónimos del eje hacen referencia a:

- TC/ Tiempo de trabajo jornada Completa.

- TP/ TiEmpo DE TRABajo JoRnada Parcial.

- DD/ Duración del contrato determinada.

- DI/ Duración del contrato indefinida. 


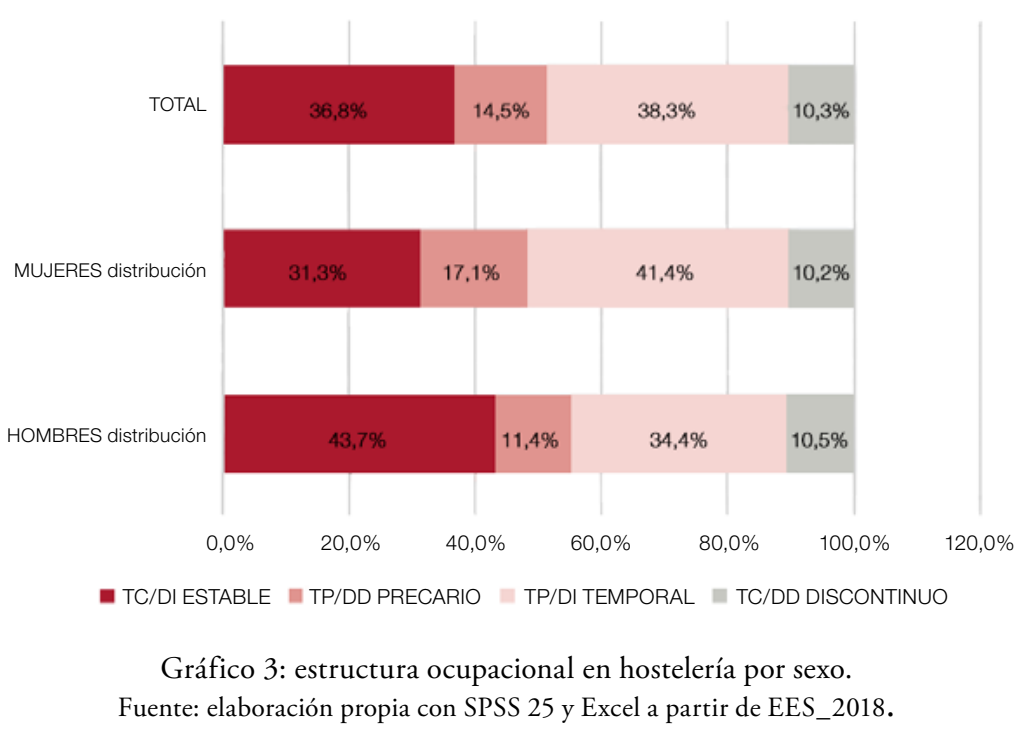

La agregación de estos indicadores da lugar a la construcción de los índices discontinuo TC/DD (10,3\%), temporal TP/DI (38,3\%), precario $T P / D D(14,5 \%)$ y estable TC/DI (36,8\%). Como podemos comprobar el trabajo estable supone solo una parte reducida de la estructura ocupacional del sector. En cuanto a contratación resalta en los resultados que algo más de la mitad de los asalariados del sector trabaja en condiciones laborales precarias y/o temporales.

En cuanto a la distribución por sexos los hombres principalmente se distribuyen en base a contratos estables en un $43 \%$ de estabilidad laboral que disminuye para las mujeres al concentrar en esta categoría un 31,3\% de efectivos laborales. En contrapartida, las mujeres mayoritariamente se agregan en torno a la contratación temporal, un $41 \%$ de mujeres se adhieren a este tipo de contratación. La precariedad y temporalidad de los contratos en hostelería puede en cierta medida explicar las brechas salariales tanto ocupacionales como de género en el sector.

\section{Mercados locales}

La distribución salarial no se presenta geográficamente homogénea en el conjunto del Estado espańol. Cada NUTS1 poblacional presenta salarialmente características específicas distintivas que nos permiten aventurar la existencia de diferentes mercados locales retributivamente heterogéneos en el sector de la hostelería. En este sentido cabe destacar que el diferencial salarial entre los NUTS 1 Noroeste y Canarias en los que el diferencial de salario mensual medio (recor- 


\begin{tabular}{|c|c|c|c|c|c|c|c|c|}
\hline \multicolumn{9}{|c|}{ TABLA 7. DISTRIBUCIÓN GEOGRÁFICA SALARIAL EN ESPAÑA } \\
\hline SALMES & & NOROESTE & NORESTE & MADRID & Centro & Este & SuR & CANARIAS \\
\hline Media & & $924,29 €$ & $1090,01 €$ & $1099,72 €$ & $927,47 €$ & $1229,40 €$ & $987,37 €$ & $1364,63 €$ \\
\hline \multirow{2}{*}{$\begin{array}{l}95 \% \text { de } \\
\text { intervalo de } \\
\text { confianza } \\
\text { para la media }\end{array}$} & $\begin{array}{l}\text { Límite } \\
\text { inferior }\end{array}$ & 899,93 & 1059,79 & 1075,00 & 900,88 & 1210,84 & 966,00 & 1339,91 \\
\hline & $\begin{array}{l}\text { Límite } \\
\text { superior }\end{array}$ & 948,65 & 1120,23 & 1124,45 & 954,06 & 1247,96 & 1008,74 & 1389,36 \\
\hline \multicolumn{2}{|c|}{ Media recortada al 5\% } & 908,92 & 1058,04 & 1042,56 & 901,56 & 1167,59 & 938,02 & 1345,03 \\
\hline \multicolumn{2}{|l|}{ Mediana } & 987,82 & 1071,39 & 1080,13 & 884,09 & 1242,47 & 858,55 & 1379,70 \\
\hline \multicolumn{2}{|l|}{ Desviación } & 464,39 & 606,15 & 678,08 & 513,01 & 774,90 & 652,97 & 557,33 \\
\hline \multicolumn{2}{|l|}{ Mínimo } & 40,33 & 11,25 & 54,19 & 99,72 & 24,40 & 70,06 & 117,85 \\
\hline \multicolumn{2}{|l|}{ Máximo } & 4130,57 & 5819,95 & 8270,52 & 6503,47 & 14715,47 & 10482,58 & 7831,60 \\
\hline \multicolumn{2}{|l|}{ Rango } & 4090,24 & 5808,70 & 8216,33 & 6403,75 & 14690,64 & 10412,52 & 7713,75 \\
\hline \multicolumn{2}{|c|}{ Rango intercuartil } & 679,73 & 905,28 & 710,68 & 642,49 & 857,80 & 958,73 & 418,52 \\
\hline \multicolumn{2}{|l|}{ Asimetría } & 0,38 & 0,99 & 2,46 & 1,66 & 2,91 & 1,47 & 1,68 \\
\hline \multicolumn{2}{|l|}{ Curtois } & 0,66 & 3,47 & 14,61 & 11,27 & 23,22 & 6,23 & 12,14 \\
\hline
\end{tabular}

Fuente: elaboración propia con SPSS 25 a partir de EES_2018.

tado al 5\%) se sitúa en torno a los $450 €$ a favor del mercado canario. Estas distribuciones salariales tan diversas, en las que no solo el indicador de salario medio revela rasgos diferenciales, proponen profundizar en cada una de las distribuciones segmentadas por NUTS1 para plantear un acercamiento más preciso a la estructura salarial de España (tabla 7).

Cabe reseñar que se dan los salarios mensuales más altos en el caso de CANARIAS y NUTS1 Este (Cataluña, Valencia y Baleares). Ambos potentes mercados turísticos donde la industria hostelera cuenta con una mayor especialización productiva. Por el contrario, en los casos de los NUTS1 Noroeste y Centro los salarios descienden considerablemente debido posiblemente al efecto contrario; menor especialización productiva turística (gráfico 4).

Precisemos; los resultados del ANOVA para todos los indicadores salariales con respecto a los grupos NUTS1 resultan significativos, por tanto y al igual que en casos anteriores, con una probabilidad bastante alta de acertar las diferencias salariales entre NUTS 1 no se deben al azar. Se crean 5 subgrupos homogéneos resultantes de implementar el estadístico HSD de Tukey (tabla 8). Estos subconjuntos comparten similares características en cuanto a medias salariales mensuales resultando en consecuencia salarialmente divergentes del resto. El primer subconjunto de características salariales homogéneas agrupa Noroeste y Centro, el segundo Centro y Sur, Noreste y Madrid, el tercero y último clúster que conforman subgrupos homogéneos propios lo compones los NUTS1 de Canarias y Este. 


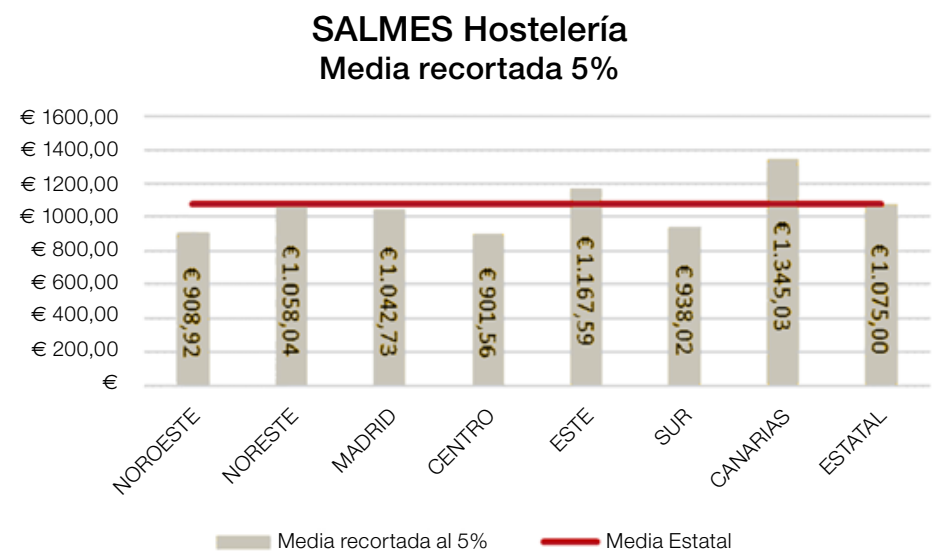

Gráfico 4: distribución estatal del salario mes en hostelería.

Fuente: elaboración propia con SPSS 25 a partir de EES_2018.

\begin{tabular}{|c|c|c|c|c|c|c|c|}
\hline \multicolumn{8}{|c|}{ TABLA 8: SUBCONJUNTOS HOMOGÉNEOS DE TUKEY. SALARIO MES } \\
\hline \multicolumn{8}{|c|}{ SALMES } \\
\hline & & \multicolumn{6}{|c|}{ SUBCONJUNTO PARA ALFA $=0.05$} \\
\hline & NUTS 1 & $\mathrm{~N}$ & 1 & 2 & 3 & 4 & 5 \\
\hline \multirow{8}{*}{$\begin{array}{l}\text { HSD } \\
\text { Turkey }\end{array}$} & Noroeste & 1398 & 924.2940 & & & & \\
\hline & Centro & 1432 & 927.4715 & 927.4715 & & & \\
\hline & Sur & 3589 & & 987.3689 & & & \\
\hline & Noreste & 1547 & & & 1090.0091 & & \\
\hline & $\begin{array}{l}\text { Comunidad } \\
\text { de Madrid }\end{array}$ & 2891 & & & 1099.7249 & & \\
\hline & Este & 6700 & & & & 9.4022 & \\
\hline & Canarias & 1953 & & & & & 1364.6341 \\
\hline & Sig. & & 1.000 & .058 & .999 & 1.000 & 1.000 \\
\hline
\end{tabular}

Se visualizan las medias para los grupos en los subconjuntos homogéneos.

a. Utiliza el tamañó de la muestra de la media armónica = 2092,873.

b. Los tamańos de grupo no son iguales. Se utiliza la media armónica de los tamaños de grupo. Los niveles de error de tipo I no están garantizados.

Fuente: elaboración propia con SPSS 25 a partir de EES_2018.

\section{EDAD Y SEXO}

En esta sección examinamos la composición de efectivos laborales del sector en cuanto a la edad y el sexo de los asalariados que la componen. Tras los descriptivos se realiza una aproximación al salario medio mensual de cada grupo de edad 


\begin{tabular}{|c|c|c|c|c|c|}
\hline \multicolumn{6}{|c|}{ TABLA 9. FRECUENCIA Y PORCENTAJES ASALARIADOS EN HOSTELERÍA } \\
\hline \multicolumn{6}{|c|}{ Sexo } \\
\hline & & Frecuencia & Porcentaje & Porcentaje VÁlido & PorCentaje ACUMUlado \\
\hline \multirow{3}{*}{ Válido } & Hombre & 518379 & 44,4 & 44,4 & 44,4 \\
\hline & Mujer & 649995 & 55,6 & 55,6 & 100,0 \\
\hline & Total & 1168374 & 100,0 & 100,0 & \\
\hline
\end{tabular}

Fuente: elaboración propia con SPSS 25 a partir de EES_2018.

\begin{tabular}{lcc}
\hline \multicolumn{1}{c}{ TABLA 10. FEMINIZACIÓN Y COHORTES DE EDAD DE LOS ASALARIADOS EN HOSTELERÍA } \\
\hline Menos de 19 & DifERENCIA EFECTIVOS & FEMINIZACIÓN \\
\hline De 20 a 29 & 3582 & $42 \%$ \\
\hline De 30 a 39 & 5242 & $49 \%$ \\
\hline De 40 a 49 & -45176 & $57 \%$ \\
\hline De 50 a 59 & -45915 & $58 \%$ \\
\hline Más de 59 & -44233 & $59 \%$ \\
\hline Total & -5116 & $54 \%$ \\
\hline
\end{tabular}

Fuente: elaboración propia con SPSS 25 y Excel a partir de EES_2018.

comprobando la significatividad de las diferencias salariales entre grupos mediante el procedimiento de Análisis de la Covarianza (ANCOVA).

Los efectivos laborales asalariados del sector hostelero en el conjunto del Estado son una fuerza laboral feminizada, aunque no excesivamente. Mientras las mujeres suponen un 55,6\% de los efectivos los hombres rebajan su participación laboral asalariada al 44,4\% (tabla 9). En números absolutos trabajan en el sector 131616 mujeres más que hombres. Si en términos absolutos el sector no presenta una excesiva feminización de sus efectivos salariales si descomponemos la distribución por tramos de edad se observa cómo las cohortes a partir de los 30 años presentan una mayor concentración de activos femeninos (tabla 10).

Examinando la pirámide de población se observa una mayor concentración de mujeres en las franjas de edad centrales. En cierta medida es lógico pensar que un mercado laboral donde la inclusión de trabajadores jóvenes está muy limitada, los efectivos laborales se concentren en las cohortes de edad centrales. Desde la perspectiva de la feminización de los efectivos salariales hosteleros se observa que aumenta conforme el agregado de asalariados avanza en edad. Una posible explicación podría basarse en la subsidiariedad de los ingresos femeninos al aporte de rentas familiares. En el capítulo de conclusiones se ahondará en esta perspectiva, en esta sección seguiremos este hilo conductor para examinar los resultados que se presentan en el gráfico 5 . 


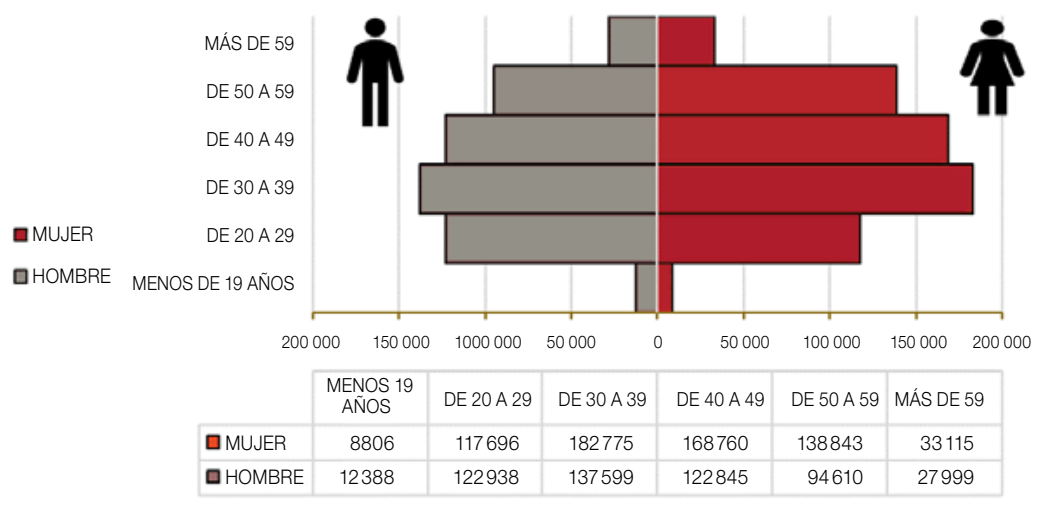

Gráfico 5: pirámide del cuerpo salarial en hostelería.

Fuente: elaboración propia con SPSS 25 y Excel a partir de EES_2018.

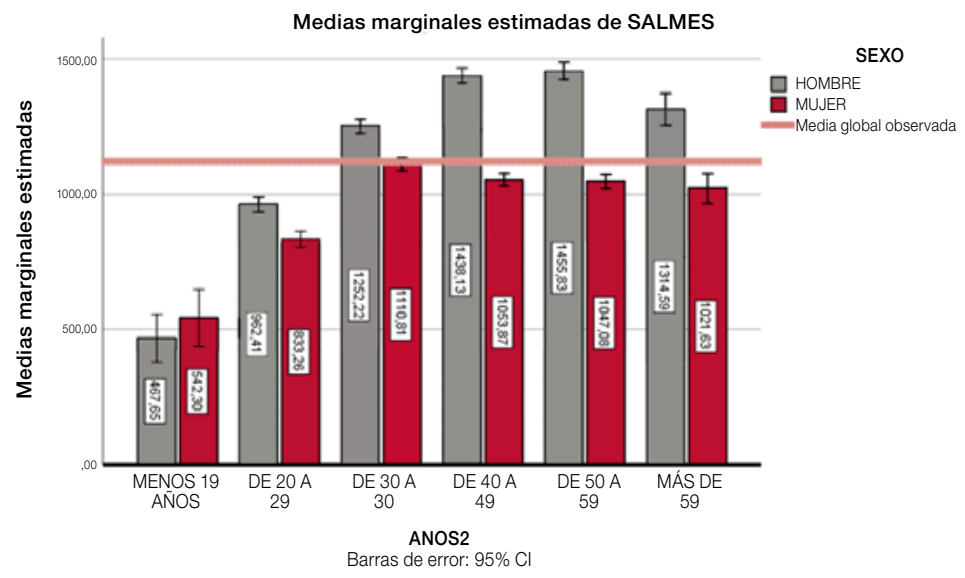

Gráfico 6: salario mes por sexo y cohortes de edad en hostelería.

Fuente: elaboración propia con SPSS 25 a partir de EES_2018.

En clave sociológica el indicador con mayores posibilidades explicativas resulta el salario mensual. Desde esta óptica acotamos el análisis de la estructura salarial al sexo y edad de los efectivos laborales al salario mes pese a que la brecha salarial de género suele calcularse en base al salario hora. En el gráfico 6 se muestran las medias estimadas para el salario mensual en hostelería por grupos de edad y sexo. Dos impresiones resaltan de los resultados: en primer lugar, la evidencia del aumento de las diferencias salariales entre sexos conforme se avanza en las cohortes de edades y, en segundo lugar, la peculiar situación de las cohortes de edades más jóvenes con respecto a las diferencias salariales entre género y el salario medio observado. 


\begin{tabular}{|c|c|c|c|c|c|}
\hline \multicolumn{6}{|c|}{$\begin{array}{l}\text { TABLA 11. SUBCONJUNTOS HOMOGÉNEOS DE TUKEY } \\
\text { POR TRAMOS DE EDAD Y SALARIO MES }\end{array}$} \\
\hline \multicolumn{6}{|c|}{ SALMES } \\
\hline & & \multicolumn{4}{|c|}{ Subconjunto } \\
\hline \multirow{8}{*}{$\begin{array}{l}\text { HSD } \\
\text { Turkey }{ }^{a, b, c}\end{array}$} & ANOS 2 & $\mathrm{~N}$ & 1 & 2 & 3 \\
\hline & Menos de 19 años & 352 & 498,1860 & & \\
\hline & De 20 a 29 & 3992 & & 899,3558 & \\
\hline & Más de 59 & 992 & & & 1160,4331 \\
\hline & De 30 a 39 & 5298 & & & 1172,0111 \\
\hline & De 50 a 59 & 3837 & & & 1213,3709 \\
\hline & De 40 a 49 & 4767 & & & 1216,7803 \\
\hline & Sig. & & 1.000 & .058 & .243 \\
\hline
\end{tabular}

El término de error es la media cuadrática (error) $=418009,566$.

a. Utiliza el tamaño de la muestra de la media armónica $=1260,870$.

b. Los tamaños de grupo no son iguales. Se utiliza la media armónica de los tamaños de grupo. Los niveles de error de tipo I no están garantizados.

c. Alfa $=, 05$.

Fuente: elaboración propia con SPSS 25 a partir de EES_2018.

Con respecto a las cohortes asalariadas más jóvenes cabe destacar, como evidencia la tabla 11, que salarialmente ambas conforman subgrupos homogéneos propios. Por una parte, está el subconjunto formado por los menores de 19 años, donde las mujeres cobran de media un salario mensual superior de aproximadamente $75 €$, y por otra el grupo de edad que abarca desde los 20 a los 29 ańos. Con respecto a este último grupo de edad reseñar que casi duplican el salario con respecto al grupo inferior en edad. Además, comienza en esta cohorte a evidenciarse la brecha salarial de género en relación con el salario mensual. A partir de los 30 años y hasta el final de la serie las cohortes de edad se agrupan homogéneamente componiendo un subconjunto propio divergente salarialmente de las cohortes más jóvenes.

Las mujeres no superan el salario medio en ninguna de las cohortes de edad mientras los hombres a partir de los 30 años comienzan a superarlos. Las mujeres alcanzan su salario mensual más alto en la cohorte de 30 a 39 años (1110€); por su parte, en los hombres el grupo de edad con mayor salario se sitúa en la cohorte que va desde los 50 a 59 años (1455€). La brecha salarial de género, en porcentaje de diferencia de ingresos entre hombres y mujeres, se sitúa en torno al $28 \%$ a partir de los 40 años hasta los 59. De otra forma explicado, las mujeres a partir de los cuarenta años mensualmente reciben salarios inferiores en un $28 \%$ con respecto a los hombres.

Para finalizar esta aproximación reseñar que el procedimiento ANCOVA, como se puede comprobar en la tabla 12, resulta significativo en cuanto a las diferencias entre medias por grupos de edad y sexo. Al igual que en anteriores secciones, en cada uno de los supuestos presentados, las diferencias entre medias de salarios 


\begin{tabular}{|c|c|c|c|c|c|c|c|}
\hline \multicolumn{8}{|c|}{ TABLA 12. ANÁLISIS DE LA COVARIANZA EDAD Y SEXO (ANCOVA) } \\
\hline \multicolumn{8}{|c|}{ Pruebas de EFECTOS INTER-SUjetos } \\
\hline \multicolumn{8}{|c|}{ VARIABLE DEPENDIENTE: SALMES } \\
\hline Origen & $\begin{array}{l}\text { TIPO III DE SUMA } \\
\text { DE CUADRADOS }\end{array}$ & gl & $\begin{array}{c}\text { Media } \\
\text { CUADRÁtICA }\end{array}$ & $\mathrm{F}$ & Sig. & $\begin{array}{c}\text { ETA PARCIAL } \\
\text { AL } \\
\text { CUADRADO }\end{array}$ & $\begin{array}{c}\text { PARÁMETRo } \\
\text { SIN } \\
\text { CENTRALIDAD }\end{array}$ \\
\hline $\begin{array}{l}\text { Modelo } \\
\text { corregido }\end{array}$ & $815376678.223^{a}$ & 11 & 74125152.566 & 177.329 & .000 & .092 & 1950.617 \\
\hline Intersección & 8010106254.038 & 1 & 8010106254.038 & 19162.495 & .000 & .499 & 19162.495 \\
\hline ANOS2 & 479255703.527 & 5 & 95851140.705 & 229.304 & .000 & .056 & 1146.519 \\
\hline Sexo & 84240975.665 & 1 & 84240975.665 & 201.529 & .000 & .010 & 201.529 \\
\hline $\begin{array}{l}\text { ANOS2b } \\
\text { Sexo }\end{array}$ & 83520380.730 & 5 & 16704076.146 & 39.961 & .000 & .010 & 199.805 \\
\hline Error & 8036651908.642 & 19226 & 418009.566 & & & & \\
\hline Total & 33063963011.502 & 19238 & & & & & \\
\hline Total corregido & 8852028586.865 & 19237 & & & & & \\
\hline
\end{tabular}

para los grupos formados por edad y sexo, con una alta probabilidad de acertar, no se deben al azar; por tanto, al menos provisionalmente, debemos rechazar la hipótesis nula de igualdad entre las medias, y aceptar la hipótesis alternativa que sugiere que estas diferencias son producto de condicionantes externos que de una forma $\mathrm{u}$ otra afectan a la estructura salarial del sector hostelero en España.

\section{CONCLUSIONES Y PROPUESTAS DE DESARROLLOS FUTUROS}

Socialmente importa la utilidad del trabajo que se ejerce, pero quizás lo que más importa es lo que se gana, o, en otras palabras, el estándar de vida que es posible permitirse con los ingresos que se reciben. Como síntesis de este trabajo sería conveniente remarcar que más que conclusiones fuertes la principal potencialidad del AED se centra en poner de relieve evidencias empíricas de estructuras de dependencia estadística de los indicadores orientadas a proponer, con garantías asentadas en un conocimiento exhaustivo de los datos, avances inductivos generadores de desarrollos teóricos, nuevos proyectos de investigación, y/o propuestas de análisis estadísticos confirmatorios.

Se observa por el análisis efectuado un perfil estructuralmente devaluado en hostelería tanto geográfica como retributivamente. Por otra parte, no solo características estructurales como las reseñadas determinan la segregación retributiva del sector, también características individuales como la edad y el género influyen en 
la existencia de una brecha salarial especialmente relevante en las cohortes de edad más jóvenes y mujeres, hechos que han sido ya detectados en la bibliografía especializada desde distintos análisis.

Además del potencial que la explotación de los microdatos que la EES_2018 brinda, cabría proponer otras dos líneas de trabajo como desarrollos futuros a partir de las evidencias avanzadas en este trabajo. Una línea de trabajo sociológica basada en la segregación ocupacional, formativa, salarial y de género del sector hostelero y otra línea de investigación de carácter econométrico y estadístico fundamentada en avanzar en el desarrollo de modelos explicativos multivariantes en clave brecha salarial.

Como se ha podido evidenciar la EES_2018 permite la desagregación por los sectores económicos clasificados en la CNACE. Esta posibilidad ofrece amplias oportunidades de desarrollo analítico no solo en investigación salarial, sino también para otras dimensiones como las formativas, de relaciones laborales u ocupacionales que por razones de espacio no se han podido cubrir en este trabajo. En este sentido y dentro del marco que nos ocupa, cabe destacar varias aproximaciones a explotar fruto de las líneas de evidencias planteadas en el artículo:

I. Salarios entre sectores. La explotación de la EES_2018 permite comparar entre sectores diferentes variables. En este epígrafe pudimos corroborar la segregación salarial del sector de la Hostelería en base a ocupar la última de las posiciones retributivas. De las aproximaciones previas desarrolladas por este equipo cabe destacar los resultados obtenidos en base al nivel de estudios realizados por los asalariados. Resulta especialmente relevante el caso de los estudios de formación profesional en turismo y su relación con el nivel salarial (Marrero-Rodríguez, R. et al., 2020). En este sentido fruto del AED se comprobó experimentalmente cómo los estudios de formación profesional eran retributivamente superiores en comparación a los devengos salariales obtenidos por asalariados con otras titulaciones formativas similares en número de años de estudio necesarios para conseguir el nivel. Se observa que los asalariados en hostelería con estudios de formación profesional son porcentualmente mínimos. Como evidencia empírica a contrastar esta línea de desarrollo futuro de investigación se presume con altas posibilidades para un diagnóstico preciso y aplicado.

II. Salarios en hostelería y mercados locales. Entre las conclusiones relevantes de este artículo destaca la existencia de la diversidad geográfica salarial en hostelería. Por lo que se propone para futuras investigaciones en base a la EES-2018 efectuar una desagregación de las muestras por NUTS1. Hemos podido comprobar cómo las divergencias salariales por NUTS1 son evidentes; por tanto, cualquier aproximación en clave estructuras ocupacionales, salariales o formativas del sector hostelero sería conveniente que se realice en orden a la desagregación de la base de datos. En este artículo tal y como estudios anteriores reflejan salarialmente se ha evidenciado la singularidad del empleo para el caso canario (Marrero-Rodríguez y González-Rodríguez, 2016). Quizás y acotando las líneas de futuros trabajos con la EES-2018 las parti- 
cularidades del modelo hostelero en las Islas Canarias supondrían una línea de trabajo interesante con amplias posibilidades analíticas de desarrollo.

III. Salarios, edady sexo. De los resultados de este epígrafe se extrae la conclusión de las diferencias salariales por edad y sexo en el sector hostelero. Si bien sobre la brecha salarial de género existe suficiente literatura de corte econométrico para que la explotación de la EES-2018 no suponga un avance significativo en el conocimiento, sí cabe destacar que la aproximación al salario mensual y/o anual añade el factor tiempo de trabajo a la estimación econométrica. Este trabajo ha comprobado las diferencias salariales con respecto al salario mes por cohorte de edad y sexo mediante el procedimiento del ANCOVA. El procedimiento empleado en este artículo, ajustado a la perspectiva exploratoria de datos, se ha centrado en el criterio concreto del análisis de la covarianza. Limitaciones propias de espacio condicionan que no se entre a valorar los resultados según modelos explicativos sociológicos mediante una explicación de las causas, consecuencias y líneas de intervención para revertir las problemáticas. Igualmente, econométricamente, no se aborda en este trabajo la brecha salarial de género en hostelería mediante modelos de estimación salarial de regresión lineal.

En base a los paradigmas interdisciplinares emergentes en la gestión de las grandes bases de datos (Robles et al., 2020) fruto del plan de investigación doctoral y desarrollo de la ponencia presentada al III Congreso SOCANTUR, se pretende con este trabajo en primer lugar poner de relieve la necesidad para la comunidad científica de la Sociología del Turismo y del Ocio y ramas anexas de conocer los recursos básicos para una explotación correcta de la EES-2018. En segundo término, se ha realizado un AED en el sector hostelero y de la restauración cuyos resultados han puesto de relieve la segregación salarial del sector, las divergencias y especificidad retributiva de cada uno de los mercados locales hosteleros en España, y las diferencias salariales entre cohortes de edad y sexo. Por último, hay que destacar que de los resultados de este trabajo más que ideas fuerza, conclusiones teóricas relevantes o resultados estimatorios robustos, se propone como síntesis final de este artículo explotar la principal de las potencialidades del AED: la puesta en marcha de nuevos proyectos de investigación que desarrollen y en su caso confirmen los patrones y estructuras de dependencia estadística que los datos evidencian. 


\section{REFERENCIAS}

Acemoglu, D. (2001). «Good jobs versus bad jobs». Journal of Labor Economics, 19(1), 1-21. https:// doi.org/10.1086/209978.

Albarracín, D. (2003). «La sociedad salarial de servicios a debate: ciclo del capital, estructura social y subjetividad obrera». Cuaderno de Relaciones Laborales, 21(2), 191-213. https://revistas. ucm.es/index.php/CRLA/article/view/CRLA0303220189A.

Almazán, A., Arribas, J. M., Camarero, L. y Vallejos, A.F. (2011). Análisis estadístico para la investigación social. $\left(1 .^{\mathrm{a}}\right)$. Garceta.

Conde-Ruiz, J.I. y Marra De Artíñano, I. (2016). «Brechas Salariales de Género en España. Las claves». Fedea, 2-10. http://documentos.fedea.net/pubs/eee/eee2018-06.pdf.

Cueto, B. y SÁnchez-SÁnchez, N. (2006). «Occupational segregation as a cause of gender-based salary differences». Working Paper. Encuentros de Economía Aplicada. Asociación Libre de Economía. Spain. https://archivo.alde.es/encuentros.alde.es/anteriores/xiiieea/trabajos/ pdf/139.pdf.

De Rada, V.D. (2002). Técnicas de Análisis Multivariante para Investigación Socialy Comercial. Ejemplos prácticos utilizando SPSS versión 11. Ra-Ma.

Escobar, M. (1999). «Análisis gráfico/exploratorio». La Muralla.

García-Martínez, J.-S. (2005). «Explotación de la Encuesta de Población Activa». Metodología de Encuestas, 7, 21-41.

Huete, R. (2014). «¿A qué se dedica la sociología del turismo en Espańa?». Revista Atlántida: Revista Canaria de Ciencias Sociales, 6, 17-32. https://www.ull.es/revistas/index.php/atlantida/ article/view/1029.

Huete, R., Brotons, M. y Sigüenza, M. (2016). «La Desigualdad Entre Mujeres y Hombres en el Sector Hostelero Español». Estudios y Perspectivas En Turismo, 25(1), 73-87. http://hdl. handle.net/10045/52396.

Instituto Nacional de Estadística (INE) (2020). Encuesta de Estructura Salarial (EES). Metodologia 2020. https://www.ine.es/metodologia/t22/meto_ees18.pdf.

Krueger, A.B. y Summers, L.H. (1988). «Efficiency Wages and the Inter-Industry Wage Structure». Econometrica, 56(2), 259. https://doi.org/10.2307/1911072.

López-Roldán, P. y Fachelli, S. (2018). Metodología de la Investigación Social Cuantitativa . Bellaterra (Cerdanyola del Vallès): Dipòsit Digital de Documents, Universitat Autònoma de Barcelona. 1. ${ }^{a}$ edición. Edición digital http://ddd.uab.cat/record/129382.

Marrero-Rodríguez, R. y González-Rodriguez, P. (2016). «La compleja realidad del empleo turístico canario», en M. Simancas y E. Parra-López (eds.), ¿Existe un modelo turistico canario? (pp. 149-172). Promotuor Turismo Canarias. https://dialnet.unirioja.es/servlet/ articulo?codigo $=5782453$.

Marrero-Rodríguez, R., Morini-Marrero, S. y Ramos-Henríquez, J.M. (2020). «Tourism jobs in demand: Where the best contracts and high salaries go at online offers». Tourism Management Perspectives, 35(June). https://doi.org/10.1016/j.tmp.2020.100721.

Martín, E. (2018). «Las Encuestas de Estructura Salarial». Índice: Revista de Estadística y Sociedad, 71, 10-14. http://www.revistaindice.com/numero71/p10.pdf. 
Martínez-Gayo, G. (2019). «¿Empleos cinco estrellas ? Reflexión sobre la precariedad laboral en la hostelería española». Revista Internacional de Turismo, Empresa y Territorio, 6, 1-15. https:// www.uco.es/ucopress/ojs/index.php/riturem/article/view/12283.

Martínez-Quintana, M.V. (1994). «Tiempo de trabajo perdido: Absentismo Laboral», en Lucas Marin, A. Sociología para la empresa. Madrid: McGrawHill, pp. 409-432.

Martínez-Quintana, V. y Martínez-Gayo, G. (2019). «Empoderamiento y desigualdad en el trabajo turístico femenino. una perspectiva comparada». Papers de Turisme, 62, 37-57. http:// www.papersdeturisme.gva.es/ojs/index.php/Papers/article/view/513.

Moreno, D., y Cañada, E. (2018). «Dimensiones de género en el trabajo turístico». Serie Informes en Contraste (vol. 4). http://www.albasud.org/publ/docs/81.pdf.

Olivares, J. (2002). «Análisis Exploratorio y Análisis Confirmatorio de Datos». Espacio Abierto, 11(2).

Ons-Cappa, M., García-Pozo, A. y Sánchez-Ollero, J.L. (2017). «Incidencia de factores personales y laborales en los salarios del sector hostelero: una visión de género». Cuadernos de Turismo, 39, 417. https://doi.org/10.6018/turismo.39.290631.

Pozo-García, A., Soria-Campos, J.-A., Sánchez-Ollero, J.-L. y Marchate-Lara, M. (2011). «Capital humano y salarios en la hostelería española: Un análisis regional». Revista de Estudios Regionales, 91, 71-95.

Ramos, V., Rey-Maquieira, J. y Tugores, M. (2002). «Análisis empírico de discriminación por razón de género en una economía especializada en turismo». Annals of Tourism Research En Español, 4(1), 239-258.

Rivera Mateos, M. (2018). «Turismo, brecha salarial y desigualdades laborales de género en espacios rurales de Andalucía (España)». Cuadernos Geográficos, 57(3), 291-312. https://doi. org/10.30827/cuadgeo.v57i3.6459.

Robles, J.M., Tinguaro-Rodríguez, J., Caballero, R. y Gómez, D. (2020). Big data para científicos sociales. Una introducción. Centro de Investigaciones Sociológicas (CIS).

Rodríguez-GonzÁlez, S. (2018). «Brechas de género en el mercado laboral: una mirada crítica ante el horizonte 2030». Atlántida. Revista Canaria de Ciencias Sociales, 9, 69-89. https://doi. org/10.25145/j.atlantid.2018.09.004.

Salvador, M. y Gargallo, P. (2003). Análisis Exploratorio de Datos. 5campus.com, Estadísitica. http://www.5campus.com/leccion/aed.

Tukey, J.W. (1997). Exploratory Data Analysis. Reading: Addison-Wesley.

Visintin, S., Elvira, M., Rodriguez, C. y Lavezzolo, S. (2019). «Un Análisis Exploratorio de los Salarios y la Estabilidad Laboral en el Sector de los Cuidados de Larga Duración en Europa». Statistical Field Theor, 53(9), 1689-1699. 


\section{ANEXO 1: COMANDOS DE SINTAXIS}

* Comandos de sintaxis. Ponderación, variables derivadas y crear base de datos para Hostelería con la EES_2018.

* Debemos tener en cuenta que la ejecución de las operaciones debe realizarse en el orden que proponemos en el fichero de sintaxis pues, como por otra parte resulta lógico, para obtener las variables de ganancia deben de haberse creado anteriormente variables de tiempo de trabajo. Como el lector podrá observar los comandos de sintaxis en SPSS y aplicaciones similares permiten comentarios añadiendo al inicio el símbolo de asterisco $\left(^{*}\right)$.

COMPUTE PONDERA=FACTOTAL*216726/12976074.

VAR LAB PONDERA 'FACTOR DE PONDERACIÓN MUESTRAL'.

WEIGHT PONDERA.

FREQUENCIES VARIABLES=REGULACION

/ORDER=ANALYSIS.

* Días del mes de octubre por los que se ha percibido salario.

COMPUTE DIASMES=DRELABM-DSIESPM2.

* Días de relación laboral en el año.

COMPUTE DIASRELABA=DRELABAM*30.42+DRELABAD.

* (para ajustar, si bien no es necesario ejecutar las instrucciones IF DIASRELABA >365 THEN DIASRELABA =365).

* Días del año por los que se ha percibido salario.

COMPUTE DIASANO=DIASRELABA-DSIESPA2-DSIESPA4.

* Para el cálculo de los componentes del salario mensual.

COMPUTE SALBASE $=(31 / \text { DIASMES })^{*}$ SALBASE.

COMPUTE COMSAL $=(31 /$ DIASMES $) *$ COMSAL .

COMPUTE COMSALTT=(31/DIASMES)* COMSALTT.

* Salario mensual total (incluye pagos extraordinarios y pagos por horas extra).

COMPUTE SALMES=SALBASE+COMSAL+EXTRAORM+PHEXTRA.

* Salario mensual ordinario (no incluye pagos extraordinarios).

COMPUTE SALMOR=SALBASE+COMSAL+PHEXTRA.

* Salario mensual neto.

COMPUTE SALNETO=SALMES-COTIZA-IRPFMES.

* Salario anual total (incluye pagos extraordinarios).

COMPUTE

SALANUAL $=(365 /$ DIASANO $) *($ RETRINOIN+RETRIIN+VESPNOIN+VESPIN $)$.

* Salario anual ordinario (no incluye pagos extraordinarios ni los pagos en especie).

COMPUTE SALAOR=(365/DIASANO $)^{*}($ RETRINOIN+RETRIIN $)-$ GEXTRA. 
* Para el cálculo de los componentes del salario anual. COMPUTE VESPNOIN=(365/DIASANO)*VESPNOIN.

COMPUTE VESPIN $=(365 / D I A S A N O)^{*}$ VESPIN.

COMPUTE GEXTRA=(365/DIASANO $)^{*}$ GEXTRA.

* Horas normales de trabajo en el mes de octubre.

COMPUTE JMP1 $=(J S P 1+J S P 2 / 60) * 4.35+$ HEXTRA.

* Ganancia por hora normal de trabajo.

COMPUTE SALHORA=SALMES/JMP1.

WEIGHT OFF.

DATASET NAME ConjuntoDatos1 WINDOW=FRONT.

RECODE CNACE ('IO'=1) (ELSE=SYSMIS) INTO EESHOSTEL.

VARIABLE LABELS EESHOSTEL 'Hostelería'.

EXECUTE.

DATASET COPY EESHOSTEL.

DATASET ACTIVATE EESHOSTEL.

FILTER OFF.

USE ALL.

SELECT IF (NOT $($ EESHOSTEL $=0)$ ).

EXECUTE.

DATASET ACTIVATE ConjuntoDatos1.

DATASET ACTIVATE EESHOSTEL. 
